\title{
Development of Real Time Wave Simulation Technique
}

\author{
by Ben T. Nohara, Member łkuo Yamamoto, Member \\ Masami Matsuura, Member
}

\begin{abstract}
Summary
The research of ocean waves has been important, not changing since the Age of Great Voyages, because we can't still get the complete knowledge of them. The objective of this research is to develop the fast calculation process of ocean waves, which includes the customized hardware as well as the software algorithm. The authors have realized the real time simulation of many types of ocean waves by the parallel computing system which consists of a PC (Personal Computer) and DSPs (Digital Signal Processor). The real time wave synthesis executing in DSPs is also developed. The methods are applied to a wave simulator as well as a wave maker.
\end{abstract}

\section{Introduction}

This paper describes the real time simulation technique of ocean waves, so-called short crested waves, which are characterized by directional spectra.

In recent years, the necessity of the studies on extreme environmental loads acting on ships as well as offshore structures has been pointed out, because the demand of structural safety of floating structures in severe storm conditions has been increasing. So, the computer simulation of ocean waves is important.

However, the time consuming process is required to simulate real ocean waves even by a quite large computer. Therefore, the authors have developed the customized hardware and its associated software for real time animation of ocean waves.

The hardware of the developed system is structured by so-called a parallel computing system which consists of a PC and a wave calculation unit installed by DSPs. To achieve real time animation, the algorithm of real time wave calculation is developed.

The paper presents the formulation of ocean waves, the hardware configuration of the developed system, and the real time algorithm of simulation.

\section{Formulation of Ocean Waves}

In the wave equations the water surface elevation of a fundamental wave is formulated as a sinusoidal function ${ }^{1-6)}$. Therefore irregular waves of the ocean

\section{Mitsubishi Heavy Industries, LTD.}

Received 10 th July 1996

Read at the Autumn meeting 14, 15 th Nov. 1996 can be considered as a linear superposition of a large number of sine (or cosine) waves all traveling independently of one another in different directions with different frequencies.

The spectral analysis based on linear theory describes how energy of each wave is distributed in direction and frequency.

Let $(x, y)$ and $t$ be the horizontal plane of ocean and time, then the water surface elevation of the ocean $\eta(x$, $y, t)$ is generally written by

$$
\begin{aligned}
\eta(x, y, t)= & \sum_{n=1}^{N} \sum_{m=1}^{M} a_{n m} \cos \left(K_{n} x \cos \theta_{m}\right. \\
& \left.+K_{n} y \sin \theta_{m}+\sigma_{n} t+\varepsilon_{n m}\right),
\end{aligned}
$$

where the subscription $n$ and $m$ indicate the values of $n$ -th frequency and $m$-th direction, respectively, and $N$, $M, a_{n m}, K_{n}, \theta_{m}, \sigma_{n}, \varepsilon_{n m}$ denote the number of component waves in frequency, the number of component waves in direction, the amplitude of $n m$-th component wave, the wave number of $n$-th frequency wave, the $m$-th wave propagation angle, the angular frequency of $n$-th frequency wave, and the random phase lag defined from 0 to $2 \pi$, respectively.

In equation ( 1 ), the wave number of $K_{n}$ must satisfy $\sigma_{n}^{2}=g K_{n} \tan ^{-1} K_{n} h$,

where $g$ and $h$ denote the acceleration due to gravity and the depth of ocean bed, respectively, which is called the dispersion relation. The amplitude of $a_{n m}$ is represented by

$$
a_{n m}=\sqrt{2 S_{\eta}\left(\sigma_{n}, \theta_{m}\right) \Delta \sigma_{n} \theta_{m}},
$$

where $S_{\eta}$ denotes directional spectrum which is given by

$$
S_{\eta}(\sigma, \theta)=S(\sigma) G(\theta),
$$

$S(\sigma)$ denotes one dimenional ocean wave spectrum which is proposed by the international committee in the 


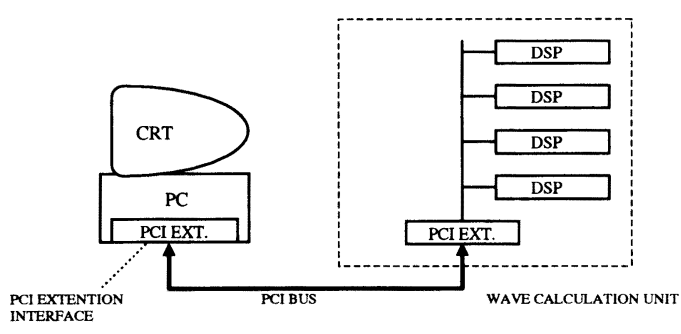

Fig. 1 Hardware Configuration of Real Time Wave Animation.

continuous form such as JONSWAP ${ }^{2)}, \mathrm{ISSC}^{3)}$, and Bretschneider-Mitsuyasu' ${ }^{4}$, etc. (see APPENDIX) $G(\theta)$ denotes directional distribution function which is represented by

$$
\begin{aligned}
& G(\theta)=\frac{\Gamma(\nu+1)}{\sqrt{\Pi \Gamma\left(\nu+\frac{1}{2}\right)}} \cos ^{2 \nu}\left(\theta-\theta_{0}\right), \\
& 0 \leqq \theta \leqq \Pi, \nu=1,2,3, \cdots
\end{aligned}
$$

where $\nu$ and $\theta_{0}$ mean the index number of the directional concentration and mean wave direction, respectively.

\section{Hardware Configuration}

Fig. 1 shows the hardware configuration of the developed system, which is so-celled a tight-coupled parallel computing system. The system consists of a PC installed by Pentium $(200 \mathrm{MHz})^{7)}$ and a wave calculation unit which is connected with a PC through a PCI (Peripheral Component Interconnect) ${ }^{7)}$ bus interface. The wave calculation unit consists of four DSP (Digital Signal Processor) units, and a PCI bus interface. TMS $320 \mathrm{C} 30^{8)}$ is installed in the DSP unit.

Under this system the tasks of the wave simulation are divided into several parts and executed simultaneously, which is described in the next section.

\section{Real Time Algorithm}

\section{1 The iterative equations}

Let equation (1) be changed to the discrete time form of equation ( 6 ). The water surface elevation $\eta_{i j k}$ of the $i j$-th segmented area of $(x, y)$ plane at the $k$-th time step is as follows :

$$
\begin{aligned}
\eta_{i j k}= & \sum_{n=1}^{N} \sum_{m=1}^{M} a_{n m} \cos \left(i K_{n} b \cos \theta_{m}\right. \\
& \left.+j K_{n} b \sin \theta_{m}+k \sigma_{n} \Delta t+\varepsilon_{n m}\right),
\end{aligned}
$$

where $b$ and $\Delta t$ denote the horizontal resolution and the sampling time of wave calculation.

Here

$$
\begin{aligned}
& \xi_{n i j}=\sum_{m=1}^{M} a_{n m} \cos \left(i K_{n} b \cos \theta_{m}+j K_{n} b \sin \theta_{m}+\varepsilon_{n m}\right) \\
& \zeta_{n i j}=\sum_{m=1}^{M} a_{n m} \sin \left(i K_{n} b \cos \theta_{m}+j K_{n} b \sin \theta_{m}+\varepsilon_{n m}\right)
\end{aligned}
$$

then

$$
\eta_{i j k}=\sum_{n=1}^{N}\left\{\xi_{n i j} \cos \left(k \sigma_{n} \Delta t\right)-\xi_{n i j} \sin \left(k \sigma_{n} \Delta t\right)\right\}
$$

The direct calculation of equation ( 9 ) is not suitable for real time because it has the calculation of $N$ times sine and $N$ times cosine. Then let $\phi_{n i j k}$ and $\Phi_{n i j k}$ be

$$
\left(\begin{array}{l}
\phi_{n i j k} \\
\Phi_{n i j k}
\end{array}\right)=\left(\begin{array}{ll}
\xi_{n i j} & -\zeta_{n i j} \\
\zeta_{n i j} & \xi_{n i j}
\end{array}\right)\left(\begin{array}{c}
\cos \left(k \sigma_{n} \Delta t\right) \\
\sin \left(k \sigma_{n} \Delta t\right)
\end{array}\right)
$$

then the following time step relation for iteration is obtained.

$$
\left(\begin{array}{l}
\phi_{n i j, k+1} \\
\Phi_{n i j, k+1}
\end{array}\right)=\left(\begin{array}{rr}
-\beta_{n} & \alpha_{n} \\
\alpha_{n} & \beta_{n}
\end{array}\right)\left(\begin{array}{l}
\Phi_{n i j k} \\
\phi_{n i j k}
\end{array}\right)
$$

Here

$$
\begin{gathered}
\alpha_{n}=\cos \left(\sigma_{n} \Delta t\right) \\
\beta_{n}=\sin \left(\sigma_{n} \Delta t\right)
\end{gathered}
$$

As the results of that, the final formula is as follows:

$$
\eta_{i j k}=\sum_{n=1}^{N} \phi_{n i j k}
$$

\section{2 Real time calculation}

First step

The water surface elevation of the $i j$-th segmented area is calculated by the following procedure. Based on the parameters of $\phi_{n i j 0}, \Phi_{n i j 0}, \alpha_{n}$, and $\beta_{n}$ at the first time step $k=1: \phi_{n i j 1}$ and $\Phi_{n i j 1}$ are obtained as

$$
\left(\begin{array}{c}
\phi_{n i j 1} \\
\Phi_{n i j 1}
\end{array}\right)=\left(\begin{array}{rr}
-\beta_{n} & \alpha_{n} \\
\alpha_{n} & \beta_{n}
\end{array}\right)\left(\begin{array}{l}
\Phi_{n i j 0} \\
\phi_{n i j 0}
\end{array}\right)
$$

from equation (11). Therefore the water surface elevation of the $i j$-th segmented area of the first time step can be calculated by

$$
\eta_{i j 1}=\sum_{n=1}^{N} \phi_{n i j 1} \text {. }
$$

The $k$-th step

The $(k-1)-t h$ step: $\phi_{n i j, k-1}$ and $\Phi_{n i j, k-1}$ are obtained from the previous stage, therefore, generally, $\phi_{n i j k}, \Phi_{n i j k}$ and $\eta_{i j k}$ are given by

$$
\begin{aligned}
& \left(\begin{array}{c}
\phi_{n i j k} \\
\Phi_{n i j k}
\end{array}\right)=\left(\begin{array}{rr}
-\beta_{n} & \alpha_{n} \\
\alpha_{n} & \beta_{n}
\end{array}\right)\left(\begin{array}{l}
\Phi_{n i j, k-1} \\
\phi_{n i j, k-1}
\end{array}\right) \\
& \eta_{i j k}=\sum_{n=1}^{N} \phi_{n i j k} .
\end{aligned}
$$

\section{3 Process of real time simulation}

It requires an enormous amount of calculation to compute the water surface elevation.

The calculation of equation ( 6 ) has the time consuming process with $M$ times sine and $M(N+1)$ times cosine. By the newest method $^{9)}$, an EWS takes 72 hours for a 20 -second calculation every other 0.1 second.

Even using a DSP, it takes over $4.8[\mu \mathrm{sec}]$ (79 cycles in TMS320C30 $33[\mathrm{MHz}]$ action) to calculate only a sinusoidal function in 32 bits floating point.

As well known, DSP is specialized to calculate multiplication or summation. To calculate a multiplication or summation takes only a cycle time (61[nsec]). Therefore to get the real time simulation of wave synthesis, the algorithm without sinusoidal function described above must be introduced.

Fig. 2 shows the flowchart of the wave simulation algorithm. To achieve real time simulation of ocean waves, the tasks are divided into three stages: the pre-calculation of the parameters $\left(\xi_{n i j}, \zeta_{n i j}, \alpha_{n}, \beta_{n}\right)$ of the 


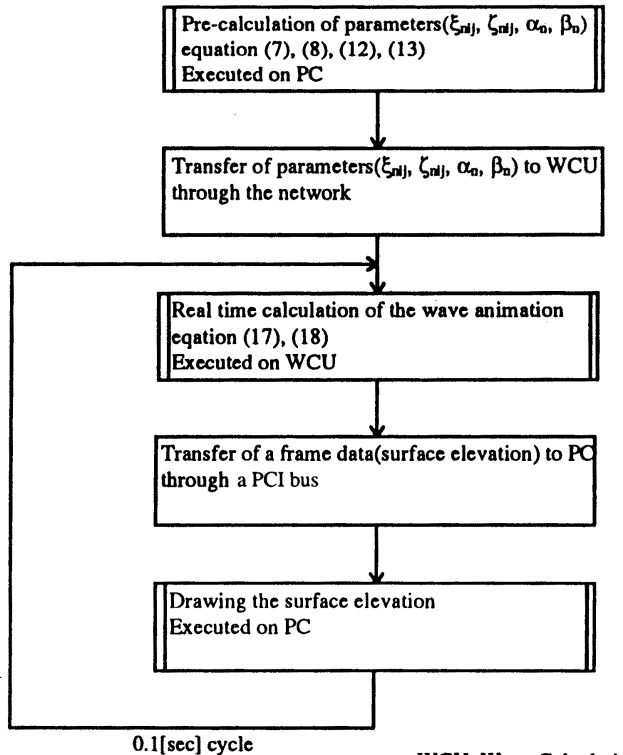

WCU: Wave Calculation Unit

Fig. 2 Flowchart of the Wave Animation Algorithm.

wave, the real time calculation of the wave synthesis, and the drawing of the surface elevation. The first stage is executed on the PC before real time calculation and drawing of the surface elevation. The second stage is executed on the wave calculation unit and the third is on the PC. The real time calculation and drawing of the surface elevation are performed every other 0.1 [sec]. Therefore the animation can be accomplished by the refresh rate of $10[\mathrm{~Hz}]$.

\section{Result}

Fig. 3, 4, and 5 show some snap shots of computer animation of real ocean waves, whose parameters are indicated in the figure's capture. These figures display the ocean area of $12.8[\mathrm{~m}] \times 12.8[\mathrm{~m}]$, in which area $(x, y)$ is divided by $128 \times 128$. Therefore the mesh of the calculation is $0.1[\mathrm{~m}]$. In this simulation the values 50 and 28 are assigned to $N$ and $M$, respectively. So, short crested waves consist of 1,400 unit waves at a point. Accordingly, the generated waves realize real ocean waves a quite well like Fig. 3, 4, and 5 .

By modifying the mesh of the calculation, the program can execute the animated picture of the ocean area ranging $10\left[\mathrm{~cm}^{2}\right]$ and $1\left[\mathrm{~km}^{2}\right]$.

The algorithm and customized hardware with a PC and DSPs developed by the authors have made it possible to display the animation of many types of real ocean waves featured by directional spectra, wave period, and wave height, etc. at the refresh rate of $10[\mathrm{~Hz}]$, in which refresh rate short crested waves are smoothly streaming.

Based on this system, the computer simulation of forces acting on the floating body, for example, ships,
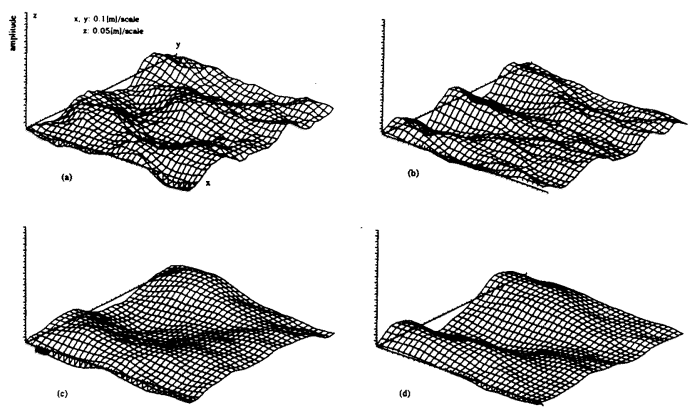

Fig. 3 Snap Shots of Computer Animation of Real Ocean Waves of JONSWAP Spectrum with its Significant Wave Height: $30[\mathrm{~cm}]$ and the crest index in equation (23): 3.3 .

(a) Peak period in equation (19): $T_{p}=$ $2[\mathrm{sec}]$

Index number of the directional concentration in equation $(5): \nu=1$

(b) Peak period in equation (19): $T_{p}=$ $2[\mathrm{sec}]$

Index number of the directional concentration in equation $(5): \nu=3$

(c) Peak period in equation (19): $T_{p}=$ 3 [sec]

Index number of the directional concentration in equation $(5): \nu=1$

(d) Peak period in equation (19): $T_{p}=$ $3[\mathrm{sec}]$

Index number of the directional concentration in equation $(5): \nu=3$
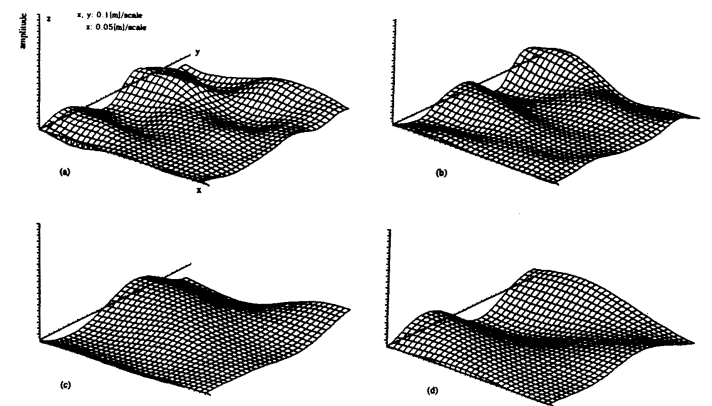

Fig. 4 Snap Shots of Computer Animation of Real Ocean Waves of ISSC Spectrum with its Significant Wave Height: $30[\mathrm{~cm}]$.

(a) Characteristic wave period in equation (25): $T=2[\mathrm{sec}]$

Index number of the directional concentration in equation $(5): \nu=3$

(b) Characteristic wave period in equation (25): $T=2[\mathrm{sec}]$

Index number of the directional concentration in equation ( 5 ): $\nu=3$

(c) Characteristic wave period in equation (25) : $T=3[\mathrm{sec}]$

Index number of the directional concentration in equation $(5): \nu=1$

(d) Characteristic wave period in equation (25): $T=3[\mathrm{sec}]$

Index number of the directional concentration in equation $(5): \nu=3$ 

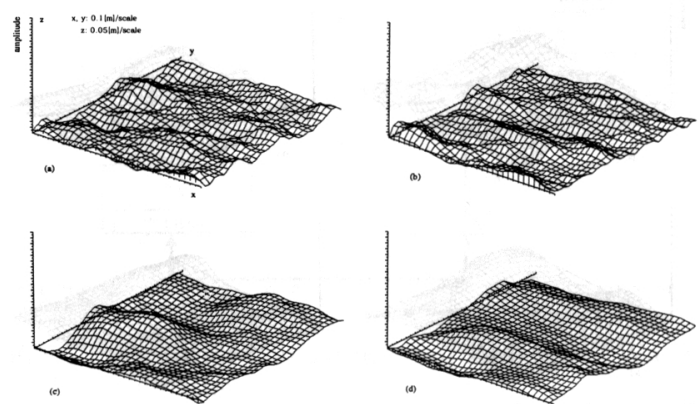

Fig. 5 Snap Shots of Computer Animation of Real Ocean Waves of Bretschneider-Mitsuyasu Spectrum with its Significant Wave Height: $30[\mathrm{~cm}]$.

(a) Significant wave period in equation (26) : $T_{1 / 3}=2[\mathrm{sec}]$

Index number of the directional concentration in equation $(5): \nu=1$

(b) Significant wave period in equation (26) : $T_{1 / 3}=2[\mathrm{sec}]$

Index number of the directional concentration in equation $(5): \nu=3$

(c) Significant wave period in equation (26) : $T_{1 / 3}=3[\mathrm{sec}]$

Index number of the directional concentration in equation $(5): \nu=1$

(d) Significant wave period in equation (26) : $T_{1 / 3}=3[\mathrm{sec}]$

Index number of the directional concentration in equation $(5): \nu=3$

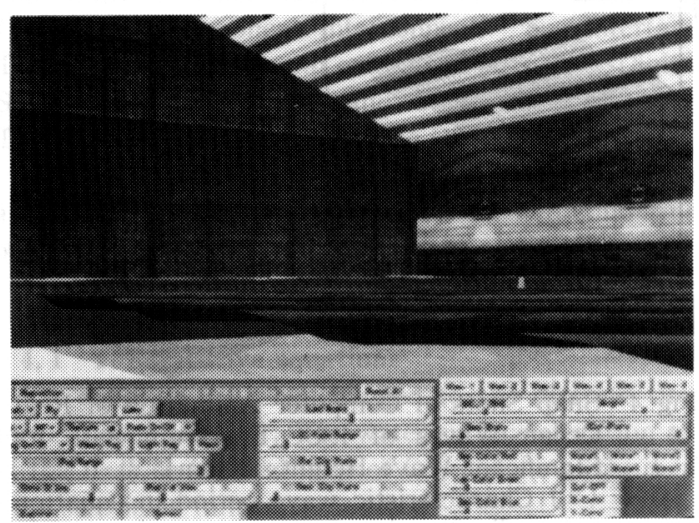

Fig. 6 Computer graphic of wave simulator for numerical tank.

harbor facilities, floating air ports, can be visualized, and then, contributed to the numerical tank development. Fig. 6 and 7 show computer graphics of wave simulator using the algorithm.

And also, the algorithm is applied to the wave genera tion of wave waker installed to the Seakeeping Basin of Nagasaki R \& D Center, Mitsubishi Heavy Industries, Ltd. shown in Fig. $8^{10)}$. The performance of the control system is summarized in the Table 1 . In the system, the

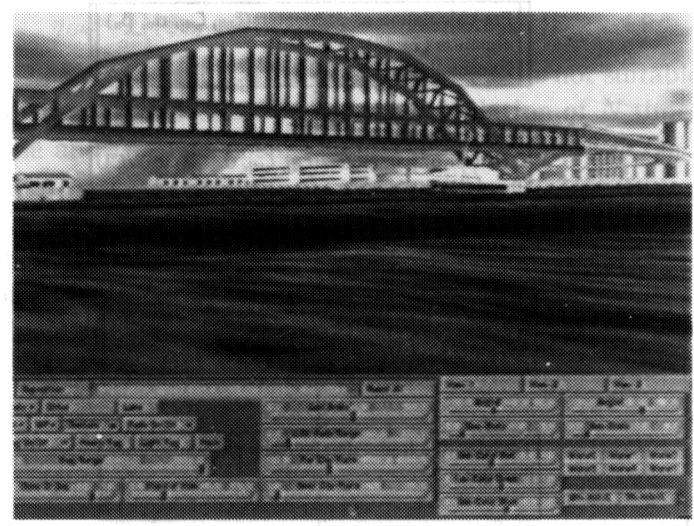

Fig. 7 Computer graphic of wave simulator for ship maneuvering.

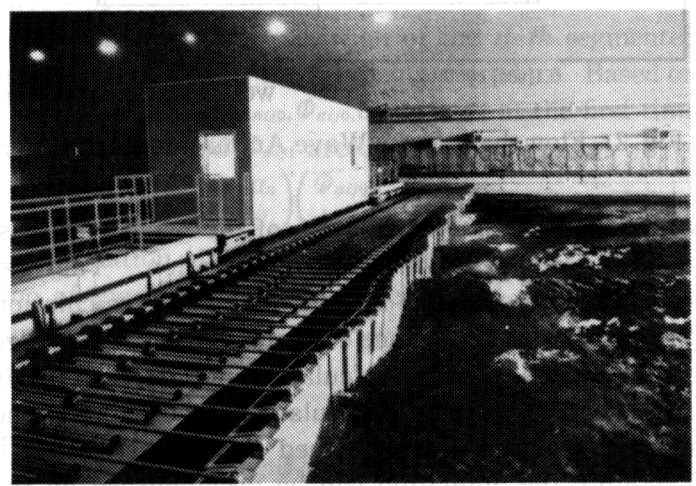

Fig. 8 Photograph of multi-directional wave waker.

Table 1 Performance of the control system

\begin{tabular}{|c|c|c|c|}
\hline & \multirow{2}{*}{$\begin{array}{l}\text { Regular waves } \\
\text { (incl. oblique) }\end{array}$} & \multicolumn{2}{|c|}{ Irregular waves } \\
\hline & & $\begin{array}{l}\text { Unidirectional waves } \\
\text { (incl. oblique) }\end{array}$ & Multi-directional waves \\
\hline Pre-calculation time & $\begin{array}{l}\text { Less than } \\
1 \mathrm{sec}\end{array}$ & $\begin{array}{l}\text { Less than } \\
3 \text { minutes }\end{array}$ & $\begin{array}{l}\text { Less than } \\
5 \text { minutes }\end{array}$ \\
\hline Control rate & $\begin{array}{r}250 \mathrm{~Hz} \\
(4 \mathrm{~ms})\end{array}$ & $\begin{array}{r}250 \mathrm{~Hz} \\
(4 \mathrm{~ms})\end{array}$ & $\begin{array}{l}250 \mathrm{~Hz} \\
(4 \mathrm{~ms})\end{array}$ \\
\hline
\end{tabular}

numbers of frequency and directional component waves are 200 and 70, respectively. High performance of the control system, which is the highest of existing wave basins in the world, is obtained for both regular waves and irregular waves.

\section{Concluding Remark}

The authors have developed the fast calculation algorithm of ocean waves. The method is successfully applied to the real time simulation of wave computer graphics, and also, to a wave maker. The real time simulation of wave simulation and the operation of wave maker can be conducted by the method. 


\section{Acknowledgement}

The authors wish to thank Mr. M. Umeda for his help of making the software and Mr. K. Morimura for his support of designing the computer graphics.

\section{Appendix}

Some spectra of ocean are proposed by the international committee in the continuous form.

JONSWAP (the Joint North Sea Wave Project)

$$
\begin{aligned}
& S(f)=\alpha H_{3}^{2} T_{p}^{-4} f^{-5} \\
& \quad \times \exp \left\{-1.25\left(T_{p} f\right)^{-4}\right\} \gamma^{\exp \left\{-\left(T_{p} f-1\right)^{2} / 2 \sigma^{2}\right\}}
\end{aligned}
$$

where $H_{\frac{1}{3}}$ and $T_{p}$ are the significant wave height and the peak period, respectively, and

$$
\begin{aligned}
& \alpha= 0.0624 /\left\{0.23+0.0336 \gamma-0.185(1.9+\gamma)^{-1}\right\} \\
& \sigma= 0.07 \text { if } f \leqq f_{p} \\
& 0.09 \text { if } f>f_{p} \text { : the peak frequency } f_{p}=1 / T_{p} \\
& \\
& \gamma=1 \sim 7 \text { : the crest index }
\end{aligned}
$$

This spectrum is for the deep sea.

ISSC (the International Ship Structures Congress) where

$$
S(f)=A f^{-5} \exp \left(-B / f^{-4}\right)
$$

$$
A=173 H \frac{2}{3} / T^{4}, B=691 / T^{4} \quad T: \text { the characteristic }
$$

wave period

This is also used for the deep sea spectrum for the test of ship maneuvering.

Bretschneider-Mitsuyasu

$$
S(f)=0.257 H_{\frac{2}{3}} T_{\frac{1}{3}}\left(T_{\frac{1}{3}} f\right)^{-5} \exp \left\{-1.03\left(T_{\frac{1}{3}} f\right)^{-4}\right.
$$

where $T \frac{1}{3}$ means the significant wave period. This spectrum is useful for the coastal engineering of the shallow sea.

\section{References}

1) Crapper, G. D., "Introduction to Water Waves," Ellis Horwood Ltd., (1984).

2) Hasselmann, K., et al., "Measurement of windwave growth and swell decay during the Joint North Sea Wave Project (JONSWAP)," Deuts-

- che Hydrograph, Z., No.12 (1973).

3) Huang, N. E., Tung, C., and Long, S. R., "Wave Spectra, Ocean Engineering Science," The Sea, Volume 9, Part A, p 197-237, (1990).

4) Mitsuyasu, W., "Development of Spectra of Wind and Waves (2)," in Proceedings of the 17 th coastal engineering conference, p 1-7. (1970).

5) Tucker, M. J., "Waves in Ocean Engineering," Ellis Horwood Ltd. (1991).

6) Wiegel, R. L., "Oceanographical engineering," Prentice Hall, Englewood Cliffs, New Jersey. (1964).

7) Intel, "Microprocessors," Vol. III. (1996).

8) Texas Instruments, "TMS 320 C 3 X Users Manual." (1994).

9) Maeda, H., et al., "Application of Walsh Function to Multi-Directional Ocean Wave Simulation," Journal of Soc. of Naval Arch. of Japan, Vol.177, p 187-195. (1995).

10) Nohara, B. T., Yamamoto, I., Matsuura, M. "Multi-Directional Wave Maker and its Real Time Wave Control System applied to a Seakeeping Model Basin", Proc. Wave Generation 95, p 175-194, (1995). 